\title{
A CHARACTERIZATION OF STRICTLY CONVEX BANACH SPACES
}

\section{STRAWTHER AND S. GUDDER}

ABSTRACT. A very short proof is given for Petryshyn's characterization of strictly convex Banach spaces [2].

Let $X$ be a real Banach space. Now $X$ is strictly convex (s.c.) iff every $f \in X^{*}$ attains a maximum on at most one point of the unit sphere [1]. A duality mapping is a function $J: X \rightarrow 2^{X^{*}}$ which satisfies $J(x)=\left\{f \in X^{*}\right.$ : $f(x)=\|f\|\|x\|$ and $\|f\|=\|x\|\}$. We say that $J$ is strictly monotone if for every $x \neq y$ and every $f \in J(x), g \in J(y)$ we have $(f-g)(x-y)>0$ [2]. For $f \in$ $J(x), g \in J(y)$, we see from the following expansion due to Browder that

$$
(f-g)(x-y)=(\|x\|-\|y\|)^{2}+(\|f\|\|y\|-f(y))+(\|g\|\|x\|-g(x)) \geq 0 .
$$

Theorem (Petryshyn), $X$ is s.c. iff $J$ is strictly monotone.

Proof. Since each term of Browder's expansion is nonnegative we have: $J$ is not strictly monotone $\Leftrightarrow \exists x \neq y, f \in J(x), g \in J(y)$ with $(f-g)(x-y)=0 \Leftrightarrow \exists f \in X^{*}$ with

$$
f(x /\|x\|)=f(y /\|y\|)=\|f\|=\|x\|=\|y\|
$$

$\Leftrightarrow \exists f \in X^{*}$ that attains a maximum at two points of the unit sphere.

\section{REFERENCES}

1. N. Dunford and J. T. Schwartz, Linear operators. I: General theory, Pure and Appl. Math., vol. 7, Interscience, New York and London, 1958. MR 22 \#8302.

2. W. Petryshyn, A characterization of strict convexity of Banach spaces and other uses of duality mappings, J. Functional Analysis 6 (1970), 282-291. MR 44 \# 4496.

Received by the editors April 18, 1974.

AMS (MOS) subject classifications (1970). Primary 46B10; Secondary 47H05.

Key words and phrases. Banach spaces, strict convexity, duality mappings.

DEPARTMENT OF MATHEMATICS, UNIVERSITY OF DENVER, DENVER,COLORADO 80210 